\title{
Reliability and Delay Analysis of Slotted Anycast Multi-hop Wireless Networks Targeting Dense Traffic IoT Applications
}

\author{
Author1, Author2, Author3
}

\begin{abstract}
Studies on IEEE 802.15.4 MAC in the current literature for anycast multi-hop networks do not capture a node's behaviour accurately. Due to the inaccurate modeling of state-wise behaviour of a node, the optimization of network parameters has not been efficient so far. In this work, we include the state-wise behaviour of a relay node into a 3D Markov model to more accurately investigate the protocol performance. Performance analysis of the proposed analytical model is evaluated for different variants of active state length, packet length and wake up rates considering reliability and delay as key performance metrics. Performance analysis shows that the model captures the behaviour of relay nodes most accurately.
\end{abstract}

Keywords-Anycast clustered multi-hop network, 3D Markov chain, Analytical model of IEEE 802.15.4 MAC.

\section{INTRODUCTION}

Analytical models for IEEE 802.15.4 MAC proposed in the literature [1], [2] \& [3] do not accurately capture the state-wise behaviour of a relay node with generic routing strategy for large dense networks. In [1], a twodimensional Markov model for IEEE 802.15.4 MAC with anycast routing is proposed and in [2], a threedimensional Markov model for IEEE 802.15.4 multihop scenario with reduced sensing and non-homogenous traffic is analyzed. The analytical study proposed in [3] incorporates joint sleep and contention control guaranteeing throughput and SINR requirements for extending network life time. The effect of adaptive MAC parameters on single hop and multi-hop wireless sensor networks are well studied using three-dimensional Markov models in [4] and [5] respectively. This work primarily investigates the accuracy of a three-dimensional Markov chain model for slotted IEEE 802.15.4 CSMA/CA MAC with slot-wise state modelling for Sleep, Idle-Listen and Active-Tx states of a node. Our analysis concludes that node wake up rates and active state time periods have a significant impact on the performance of the network. The rest of this letter is organized as follows. Section

Author1, Author2 and Atuthor3 are with Department of Electrical Engineering, Affiliation1

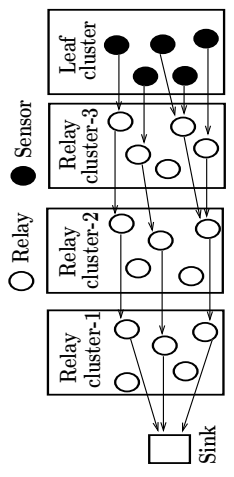

(a)

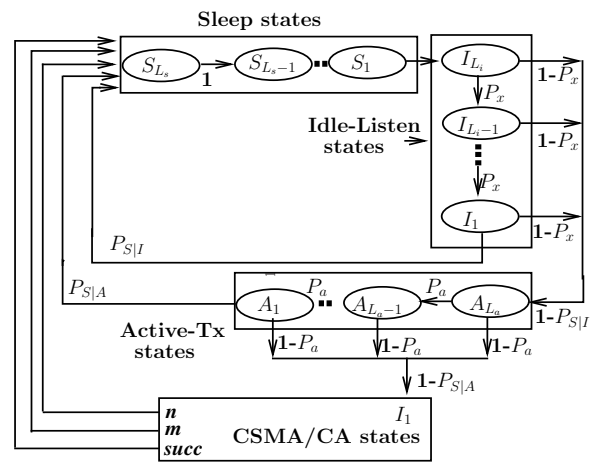

(b)
Fig. 1: a) Network scenario b) Relay node state model

2 discusses the system model based on a generic 3D Markov chain and Section 3 provides the mathematical formulation of the proposed model. The performance analysis from the emulations and the analytical model is discussed in Section 4 and finally, Section 5 concludes the letter.

\section{SYSTEM MODEL}

We consider a generic Cyber Physical Systems (CPS) wireless network scenario with four clusters as shown in Fig. 1(a). Sensor data collected at the leaf nodes have to be routed to a network sink via intermittent random relay nodes known as anycast routing. The functional model of relay nodes is captured accurately in four different states namely, Sleep $\left(S_{i}\right)$, Idle-Listen $\left(I_{i}\right)$, Active-Tx $\left(A_{i}\right)$ and CSMA/CA as shown in Fig. 1(b). In Sleep state relay nodes sleep until assigned a wake up time. In Idle-Listen state, relay nodes broadcast a beacon to the predecessor cluster (cluster-3 is the predecessor to cluster-2) and wait for a packet from it. After successfully receiving a packet in Idle-Listen state relay nodes jump to ActiveTx state and wait for a beacon from their next cluster (cluster-2 is the next cluster to cluster-3). Time intervals between beacons observed by a relay in Active-Tx are Poisson distributed which determine the waiting time in Active-Tx state described later in the delay model of this 
letter. The relay nodes that are successful in receiving a beacon within a maximum of $L_{a}$ slots of the ActiveTx state follow CSMA/CA flow depicted using a 3D Markov chain shown in Fig. 2(a) with backoff stages $(m)$, backoff counter $(k)$ and collision retries $(n)$ as the three dimensions.

Our primary investigation focuses on the effect of CSMA/CA retries in multi-hop scenarios with accurate state-wise behaviour of relay nodes. In this letter busy channel probabilities $\alpha$ and $\beta$ in Clear Channel Assessment (CCA1 and CCA2) states and channel sensing probability $\tau$ along with collision probability $P_{c}$ shown in Fig. 2(a) are derived considering the effect of Sleep, Idle-Listen and Active-Tx state probabilities along with the CSMA/CA model. With basic understanding of busy probabilities in [4], [5] and [6], one can drive the mathematical model in the following section.

\section{MAThematicAL MODEL}

We formulate the proposed model in two stages by deriving the transition probabilities for all states in Fig. 1(b) in the first stage and then follows the formulation of CSMA/CA model in second stage. In the rest of our discussion $\mu_{w}$ indicates average wake up rate per node in a cluster consisting of $N$ nodes, $L_{a}$ and $L_{i}$ are length of active and idle slots respectively.

In Eq. (1), $P_{x}$ is the transition probability of a relay node to move into the next Idle-Listen slot from the current one, when there is no packet arrivals at a given slot with an average of $\lambda$ Poisson arrivals and $P_{a}$ indicates the transition probability of a relay node to move into the next Active-Tx slot from the current, when there is no beacon arrival at a given slot with an average of $\lambda_{a}$ Poisson arrivals shown in Eq. (4). Using Eq. (1), the probability of a node transitioning to Sleep state from the last slot of Idle-Listen $\left(P_{S \mid I}\right)$ and Active-Tx $\left(P_{S \mid A}\right)$ states can be obtained as shown in Eq. (2) and (3) respectively. Finally $P_{S \mid C S M A}$ in Eq. (5) indicates the transition probability of a node from CSMA/CA state to the first slot of Sleep state which should always equal to one.

$$
\begin{gathered}
P_{x}=\exp (-\lambda) \quad, \quad P_{a}=\exp \left(-\lambda_{a}\right) \\
P_{S \mid I}=P_{S_{L_{s}} \mid I_{1}}=P_{x}^{L_{i}} \\
P_{S \mid A}=P_{S_{L_{s}} \mid A_{1}}=P_{a}^{L_{a}} \\
\lambda_{a}=\mu_{w} * N \\
P_{S \mid C S M A}=P_{S_{L_{s}} \mid C S M A_{m}}+P_{S_{L_{s}} \mid C S M A_{n}} \\
+P_{S_{L_{s}} \mid C S M A_{\text {succ }}}=1 \\
P_{S_{0}}=P_{S_{0}} P_{x}^{L_{i}}+P_{S_{0}}\left(1-P_{x}^{L_{i}}\right) P_{a}^{L_{a}}+P_{S \mid C S M A} b_{0,0,0}
\end{gathered}
$$

In Eq. (5), $P_{S_{L_{s}} \mid C S M A_{m}}$ and $P_{S_{L_{s}} \mid C S M A_{n}}$ are the probabilities to enter into the first slot of Sleep state when the received packet is discarded in CSMA/CA after exceeding maximum $m$ and $n$ respectively. $P_{S_{L_{s}} \mid C S M A_{\text {succ }}}$ is the probability of the node to enter the first slot of Sleep state after the node successfully forwards the packet. Eq. (6) can be simplified to arrive at probability $P_{S_{0}}$ of a node to stay in the first sleep slot at any given time slot in terms of $b_{0,0,0}$, where $b_{0,0,0}$ is the probability of a node to stay in the first CCA1 slot of the CSMA/CA model.

$$
\begin{array}{r}
P_{C S M A}=\sum_{i=0}^{m} \sum_{k=0}^{W_{i}-1} \sum_{j=0}^{n} b_{\mathrm{i}, \mathrm{k}, \mathrm{j}}+\sum_{j=0}^{n} \sum_{i=0}^{m} b_{\mathrm{i}, \mathrm{i}, \mathrm{j}} \\
+\sum_{j=0}^{n}\left(\sum_{k=0}^{L_{s}-1} b_{-1, \mathrm{k}, \mathrm{j}}+\sum_{k=0}^{L_{c}-1} b_{-2, \mathrm{k}, \mathrm{j}}\right)=Z_{1} * b_{0,0,0} \\
P_{S}+P_{I}+P_{A}+P_{C S M A}=1 \\
P_{S_{0}}\left[L_{s}+\sum_{i=1}^{L_{i}} P_{x}^{i-1}+\left(1-P_{x}^{L_{i}}\right) \sum_{i=1}^{L_{a}} P_{a}^{i-1}\right] \\
+Z_{1} b_{0,0,0}=1
\end{array}
$$

The probability of a node to reside in CSMA/CA state $\left(P_{C S M A}\right)$ at a randomly given time slot is the sum of backoff, CCA2, success and failure state probabilities respectively given in Eq. (7). $Z_{1}$ indicates the proportionality factor after expressing all probabilities in Eq. (7) in terms of $b_{0,0,0}$. In Eq. (8) $P_{S}, P_{I}, P_{A}$ and $P_{C S M A}$ indicate the probabilities for a node to reside in Sleep, Idle-Listen, Active-Tx and CSMA/CA states respectively in any given random time slot. All probabilities $P_{S}, P_{I}, P_{A}$ and $P_{C S M A}$ of Fig. 1(b) can be written in terms of $P_{S_{0}}$ and $b_{0,0,0}$ as shown in Eq. 9. Now using normalization Eq. (8), $b_{0,0,0}$ can be derived as in Eq. (10). After getting the relation for $b_{0,0,0}$, we derive $\alpha, \beta$ and $\tau$ expressions utilizing $b_{0,0,0}$ in a similar methodology shown in [6]. Numerical methods are used to solve highly nonlinear $\alpha, \beta, \tau$ and $b_{0,0,0}$ to arrive at a closed form solution. Both node and cluster probabilities $\left(\alpha, \beta, \tau\right.$ and $\left.P_{c}\right)$ are in close agreement with less than $5 \%$ deviation due to equal number of nodes in each cluster and random selection of relay nodes. Reliability and delay of the proposed model are derived and analyzed in the following section.

\section{A. Reliability model}

The reliability of a relay node can be determined by deriving the failure probabilities. Failure can occur due to exceeding $m, n$ and active timeout. Considering these cases, the reliability of node $\mathrm{k}\left(R_{k}\right)$ is given in Eq. (11). 


$$
b_{0,0,0}=\frac{\left(1-P_{x}\right)\left(1-P_{a}\right)\left(1-P_{x}^{L_{i}}\right)\left(1-P_{a}^{L_{a}}\right)}{\left(1-P_{a}\right) *\left[L_{s}\left(1-P_{x}\right)+L_{i}\left(1-P_{x}^{L_{i}}\right)\right]+\left(1-P_{x}^{L_{i}}\right)\left(1-P_{a}^{L_{a}}\right)\left(1-P_{x}\right) *\left[L_{a}+Z_{1}\left(1-P_{a}\right)\right]}
$$
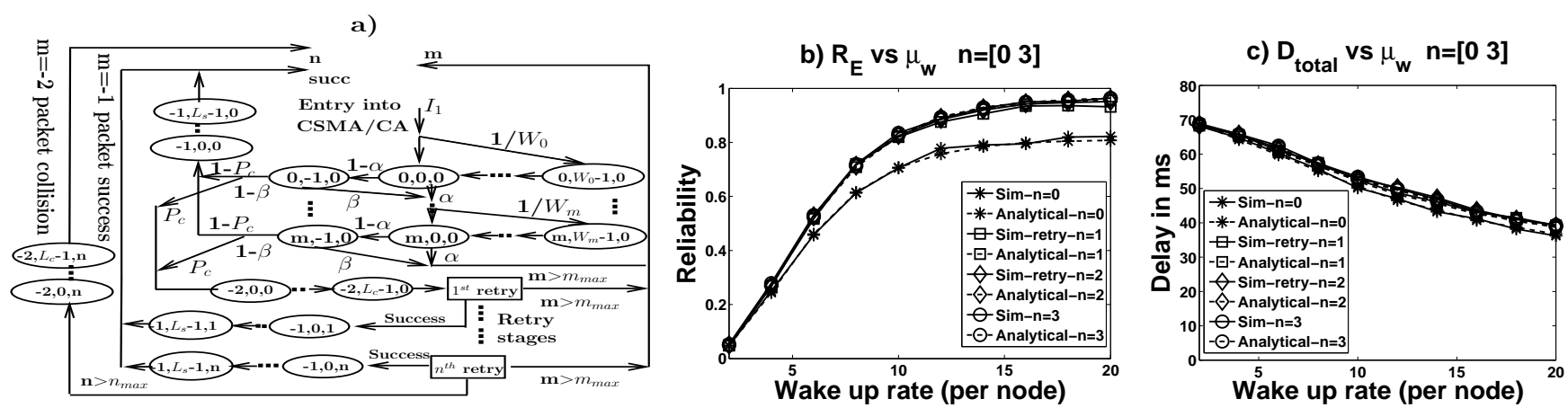

Fig. 2: 2a) Three-dimensional Markov model of IEEE 802.15.4 CSMA/CA; Fig. 2b and 2c: Simulation parameters for 2a) and 2b) are $m_{0}=3, \mathrm{~m}=4, \lambda=0.01, n=\left[\begin{array}{ll}0 & 3\end{array}\right], \mu_{w}=\left[\begin{array}{ll}2 & 20\end{array}\right], L_{a}=100, L_{p}=10, L_{i}=100, L_{s}=100$.

$R_{E}$ in Eq. (12) depicts the end-to-end reliability calculated over $h$ independent links/clusters/hops. $\widetilde{y}$ indicates the probability of a node transitioning to next retry after successfully sensing the channel from any of the $m$ stages shown in Fig. 2(a).

$$
\begin{gathered}
R_{k}=1-\left(x^{\mathrm{m}+1}(1+\widetilde{y})-\widetilde{y}^{\mathrm{n}+1}\right)\left(1-P_{a}^{L_{a}}\right)-P_{a}^{L_{a}} \\
R_{E}=\prod_{k=1}^{h} R_{k}
\end{gathered}
$$

\section{B. Delay Model}

Total delay incurred by an individual node for forwarding a packet successfully is contributed by CSMA/CA delay and active state delay. Delay due to CSMA/CA is given in Eq. (13).

$$
\begin{aligned}
& D_{\text {csma }}=T_{s}+D_{\text {avg }}+\left(T_{s}+D_{\text {avg }}\right)\left[\frac{y}{1-y}\right. \\
& \left.-\frac{\left((n+1) *\left(y^{(\mathrm{n}+1)}\right)\right)}{1-y^{\mathrm{n}+1}}\right] \\
& D_{\text {avg }}=2 S_{b}\left[1+0.25\left\{\frac { 1 - b _ { l } } { 1 - b _ { l } ^ { m + 1 } } \left[2 W_{0} \frac{1-2 b_{l}^{m+1}}{1-2 b_{l}}\right.\right.\right. \\
& \left.\left.\left.\left.-\frac{3(\mathrm{~m}+1) b_{l}^{\mathrm{m}+1}}{1-b_{l}}\right]\right]+\frac{3 b_{l}}{1-b_{l}}-\left(W_{0}+1\right)\right\}\right] \\
& P_{(\text {delay }=i) \mid \text { success }}=\frac{P_{a}^{i-1}\left(1-P_{a}\right)}{\left(1-P_{a}^{L_{a}}\right)} \\
& D_{\text {active }}=\sum_{i=1}^{L_{a}}(i) \frac{P_{a}^{i-1}\left(1-P_{a}\right)}{\left(1-P_{a}^{L_{a}}\right)} \\
& D_{\text {total }}=\left(D_{\text {csma }}+D_{\text {active }} * S_{b}\right) * h
\end{aligned}
$$

In Eq. (13), $D_{\text {avg }}$ indicates the backoff delay and is derived in Eq. (14) by taking expectation over $m$ stages of CSMA/CA, where $D_{\text {csma }}$ of a link can be obtained from computing average probability of success after $j$ retries. $T_{s}$ and $S_{b}$ are packet transmission time and unit backoff time, $b_{l}$ is $\max \{\alpha,(1-\alpha) \beta\}, W_{0}$ indicates minimum backoff window. $L_{p}$ and $L_{c}$ are length of time slots required for successful packet transmission and collision respectively and $T_{c}$ indicates packet collision time. In Eq. (16), the average number of active slots $\left(D_{\text {active }}\right)$ that a node waits before a beacon arrives is obtained. Finally total delay which is the sum of delays incurred by CSMA/CA and Active states computed over $h$ independent links is given by Eq. (17).

\section{Analytical Results}

The proposed anycast clustered multi-hop analytical model's accuracy is validated by emulating a scenario similar to that shown in Fig. 1(a) which has 4 clusters with 10 nodes each. The proposed emulation model has the following assumptions: Congestion due to ACK and interference from other $2.45 \mathrm{GHz}$ users is negligible. Each relay node switches among 3 different channels for $\mathrm{Tx}, \mathrm{Rx}$ and beacon modes to reduce interference between nearby nodes of different clusters.

We first analyze the effect of CSMA/CA retries $(n)$ on $R_{E}$ and $D_{\text {total }}$. Fig. 2(b) and Fig. 2(c) plots $R_{E}$ and $D_{\text {total }}$ versus $\mu_{w}$ respectively for 4 different values of $n$. $R_{E}$ and $D_{\text {total }}$ are observed to be increasing and decreasing respectively with increase in $\mu_{w}$ as the average waiting time to receive a beacon in active state decreases and failures in active state due to active timeout are reduced. $R_{E}$ is observed to increase by $15 \%$ and $D_{\text {total }}$ is increased 

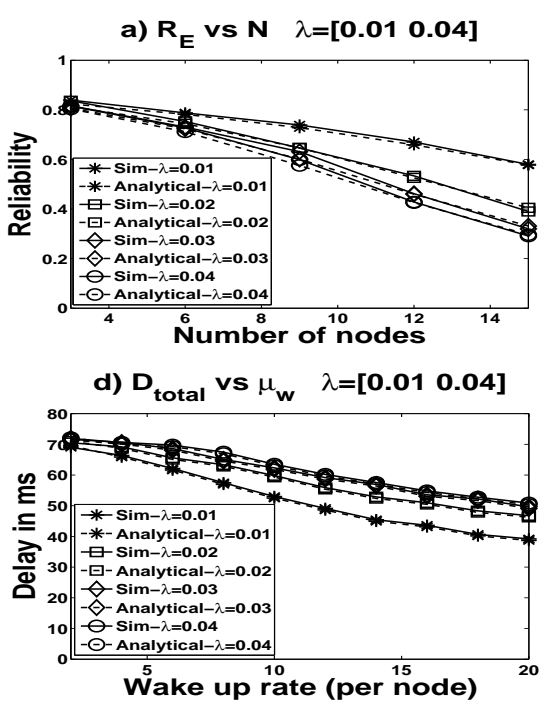

b) $D_{\text {total }}$ vs $N \quad \lambda=\left[\begin{array}{lll}0.01 & 0.04\end{array}\right]$

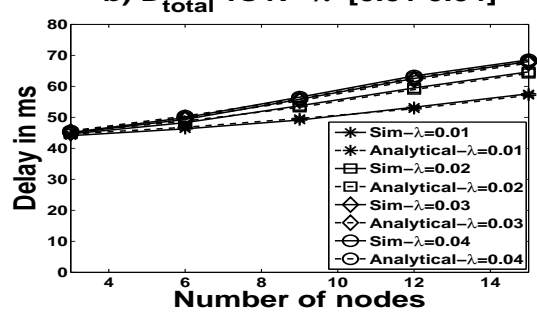

e) $R_{E}$ vs $L_{a}$ slots $\lambda=[0.010 .04]$

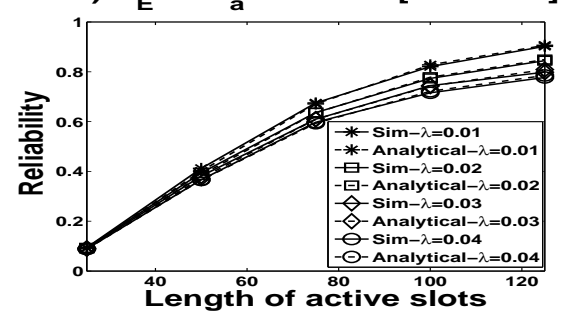

c) $R_{E} v s \mu_{w} \quad \lambda=\left[\begin{array}{ll}0.01 & 0.04\end{array}\right]$
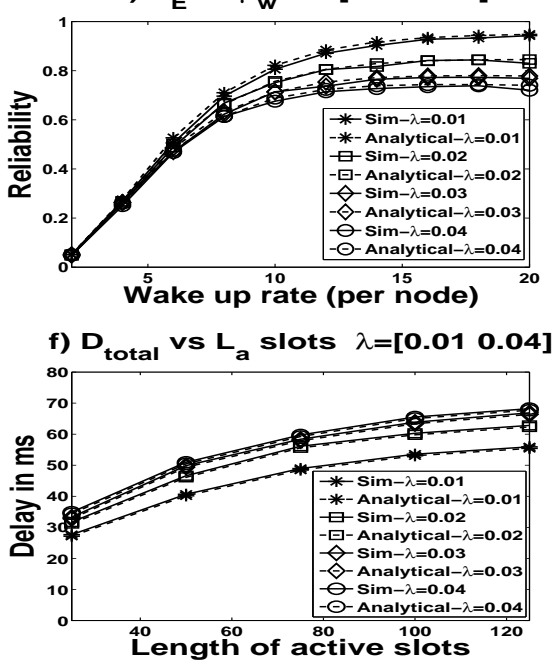

Fig. 3: Parameters for Fig. 3a and 3b: $m_{0}=3, \mathrm{~m}=4, \mu_{w}=10, \mathrm{n}=1, L_{a}=100, N=[3$ 15], $\lambda=[0.010 .04]$; Fig. $3 \mathrm{c}$ and 3d: $m_{0}=3, \mathrm{~m}=4, \mathrm{n}=1, L_{a}=100, \mu_{w}=[220], \lambda=[0.010 .04]$; Fig. 3e and 3f: $m_{0}=3, \mathrm{~m}=4, \mathrm{n}=1, L_{a}$ $=\left[\begin{array}{ll}25 & 125\end{array}\right], \lambda=\left[\begin{array}{ll}0.01 & 0.04\end{array}\right], \mu_{w}=10$; parameters fixed for all 6 scenarios: $L_{p}=10, L_{c}=10, L_{s}=100$ and $L_{i}=100$

by 6 slots with single retry after collision compared to the "no-retries" scenario. Improvement in $R_{E}$ and $D_{\text {total }}$ with higher retries ( $n=2 \& 3$ ) compared to $n=1$ is merely visible, as the probability to have successive collisions for a node is minimal. Degradation in $R_{E}$ and $D_{\text {total }}$ is observed when analysis is performed by incrementing number of nodes (N) for 4 different $\lambda$ as shown in Fig. 3(a) and 3(b). Degradation in $R_{E}$ is valid since increase in channel congestion values $(\alpha$ and $\beta$ ) results in more packet drops and collisions due to exceeding maximum $m$ and $n$. More delay $\left(D_{\text {total }}\right)$ with increase in $\lambda$ is primarily due to degradation in $D_{c s m a}$.

Fig. 3(c) and Fig. 3(d) plots $R_{E}$ and $D_{\text {total }}$ versus $\mu_{w}$ for four different $\lambda$. From the figures one can infer the importance of $\lambda$ in the performance of the network. Increase in $\mu_{w}$ reduces average waiting time in ActiveTx state and the chances for packet being dropped because of active timeout are less. The increase in $\lambda$ results in channel congestion, leading to more packet failures due to active timeout and more delay due to backoff stages.

Fig. 3(e) and Fig. 3(f) plots $R_{E}$ and $D_{\text {total }}$ versus $L_{a}$ for four different $\lambda . R_{E}$ was enhanced and $D_{\text {total }}$ was growing higher with increase in $L_{a}$ as the chances of beacon reception before active timeout increases significantly.

\section{CONCLUSiON}

In this letter, a slotted anycast model for clustered multi-hop networks with the state-wise behaviour injected into 3D Markov chain is developed and analyzed.
Reliability and delay performance metrics are analyzed with variation in parameters such as CSMA/CA retries, number of nodes, wake up rate and active time for different packet arrival values, and are validated using both analytical and emulation results with less than $0.5 \%$ error. The proposed integrated model and the analysis can greatly aid in driving the future research in modelling of dense traffic wireless multi-hop sensor networks. Optimization of model parameters is a focus of future research.

\section{REFERENCES}

[1] P. Park, C. Fischione, A. Bonivento, K. H. Johansson and A. Sangiovanni-Vincentelli, "Breath: an Adaptive Protocol for Industrial Control Applications using Wireless Sensor Networks," IEEE Transactions on Mobile Computing, vol.10, no.6, 2011.

[2] Piergiuseppe Di Marco,Pangun Park, Carlo Fischione and Karl Henrik Johansson "Analytical Modeling of Multi-hop IEEE 802.15.4 Networks," IEEE Transactions on vehicular technology, Vol. 61, no. 7, September 2012.

[3] Jae-Han Jeon, Hee-Jung Byun, and Jong-Tae Lim, "Joint Contention and Sleep Control for Lifetime Maximization in Wireless Sensor Networks," IEEE Communications Letters, vol. 17, no. 2, February 2013.

[4] P. Park, P. Di Marco, C. Fischione, and K. H. Johansson, "Modeling and Optimization of the IEEE 802.15.4 Protocol for Reliable and Timely Communications," IEEE Transactions on Parallel and Distributed Systems, vol. 24, no. 3, March 2013.

[5] Prasad, Y.R.V, Rajalakshmi P. "Analytical model of adaptive CSMA-CA MAC for reliable and timely clustered wireless multihop communication," IEEE World forum on Internet of Things (WF-IoT) conference, pp.212-217, Seoul 6-8 March 2014.

[6] S. Pollin, M. Ergen, S. C. Ergen, B. Bougard, L. Van der Perre, I. Moerman, A. Bahai, P. Varaiya, and F. Catthoor,"Performance analysis of slotted carrier sense IEEE 802.15.4 medium access layer," IEEE Transactions in Wireless Communications, vol. 7, no. 9, pp. 3359-3371, September 2008. 